\title{
Efficient and selective adsorption of neodymium on expanded vermiculite
}

\author{
Giani de Vargas Brião, Meuris Gurgel da Silva, Melissa Gurgel Adeodato Vieira*
}

School of Chemical Engineering, University of Campinas (UNICAMP), Cidade Universitária Zeferino Vaz, 13083-852, Campinas, São Paulo, Brazil

*Corresponding author.

E-mail address: melissagav@feq.unicamp.br (M.G.A. Vieira)

\section{S1: Supplementary Tables}

Table S1. Experimental domain of RCCD for the neodymium adsorption on EV.

\begin{tabular}{ccccccc}
\hline \multirow{2}{*}{ Factor } & Code & \multicolumn{5}{c}{ Levels } \\
\cline { 5 - 8 } & & $-\alpha$ & -1 & 0 & +1 & $+\alpha$ \\
\cline { 1 - 4 } particle size (mm) & $\mathrm{A}$ & 0.48 & 0.65 & 0.85 & 1.09 & 1.30 \\
adsorbent amount (g) & $\mathrm{B}$ & 0.163 & 0.300 & 0.500 & 0.700 & 0.836 \\
$\mathrm{pH}$ & $\mathrm{C}$ & 1.3 & 2.0 & 3.0 & 4.0 & 4.7 \\
\hline
\end{tabular}


Table S2. Comparison of neodymium elution process from different adsorbent matrices.

\begin{tabular}{|c|c|c|c|c|c|c|}
\hline Adsorbent & $\begin{array}{c}S / L \\
(\mathrm{~g} / \mathrm{L})\end{array}$ & $\begin{array}{l}\text { Regeneration/Elution } \\
\text { process }\end{array}$ & $\% D(\%)$ & $\begin{array}{l}\mathrm{LD}_{50} \\
(\mathrm{mg} / \mathrm{Kg})\end{array}$ & GHS class & Reference \\
\hline $\begin{array}{l}\text { Chitosan-Manganese- } \\
\text { Ferrite Magnetic } \\
\text { Beads }\end{array}$ & - & Methanol $(98 \% \mathrm{v} / \mathrm{v})$ & 90 & 1,000 & Category 4 & 1 \\
\hline $\begin{array}{l}\text { Core-shell alginate } \\
\text { beads }\end{array}$ & 1 & $\mathrm{HCl} 0.2 \mathrm{M}$ & 100 & 277 & Category 3 & 2 \\
\hline $\begin{array}{l}\text { Graphene Oxide } \\
\text { nanosheets }\end{array}$ & - & $\mathrm{HNO}_{3} 0.1 \mathrm{M}$ & $99 *$ & $>90$ & Category 3 & 3 \\
\hline $\begin{array}{l}\text { Gum Arabic grafted } \\
\text { polyacrylamide based } \\
\text { silica nanocomposite }\end{array}$ & - & $\mathrm{HCl} 0.1 \mathrm{M}$ & 80 & 277 & Category 3 & 4 \\
\hline $\begin{array}{l}\text { Ion imprinted xanthan } \\
\text { gum-layered double } \\
\text { hydroxide } \\
\text { nanocomposite }\end{array}$ & 0.025 & $\mathrm{HNO}_{3} 0.1 \mathrm{M}$ & - & $>90$ & Category 3 & 5 \\
\hline $\begin{array}{l}\text { Ethylenediaminetriac } \\
\text { etic Acid- } \\
\text { Functionalized } \\
\text { Activated Carbon }\end{array}$ & - & $\begin{array}{l}\mathrm{HCl} 0.1 \mathrm{M} \\
\text { Washing using acetone } \\
\text { and water }\end{array}$ & 99 & 277 & Category 3 & 6 \\
\hline $\begin{array}{l}\text { Acrylic Resin (110) } \\
\text { resin }\end{array}$ & 0.6 & $\mathrm{HCl} 3.0 \mathrm{M}$ & 99.4 & 277 & Category 3 & 7 \\
\hline $\begin{array}{l}\text { Expanded } \\
\text { Vermiculite }\end{array}$ & 14 & $\mathrm{CaCl}_{2} 0.5 \mathrm{M}$ & 43.2 & 1,000 & Category 4 & This work \\
\hline
\end{tabular}

*From a mixture of rare earth metals

Table S3. Selectivity of neodymium from a multicomponent solution

\begin{tabular}{lccc}
\hline Metal & $K d$ & $q(\mathrm{mmol} / \mathrm{g})$ & $S_{N d / M}$ \\
\hline $\mathrm{Ni}$ & 0.14 & 0.058 & 5.07 \\
$\mathrm{Zn}$ & 0.08 & 0.052 & 8.35 \\
$\mathrm{Cu}$ & 0.04 & 0.035 & 16.26 \\
$\mathrm{~Pb}$ & 0.02 & 0.017 & 32.26 \\
$\mathrm{Nd}$ & 0.71 & 0.065 & - \\
\hline
\end{tabular}




\section{References}

(1) Durán, S. V.; Lapo, B.; Meneses, M.; Sastre, A. M. Recovery of Neodymium (III) from Aqueous Phase by Chitosan-Manganese-Ferrite Magnetic Beads. Nanomaterials 2020, 10 (6), 1204. https://doi.org/10.3390/nano10061204.

(2) Wang, F.; Zhao, J.; Li, W.; Zhou, H.; Yang, X.; Sui, N.; Liu, H. Preparation of Several Alginate Matrix Gel Beads and Their Adsorption Properties Towards Rare Earths (III). Waste and Biomass Valorization 2013, 4 (3), 665-674. https://doi.org/10.1007/s12649-012-9179-6.

(3) Ashour, R. M.; Abdelhamid, H. N.; Abdel-Magied, A. F.; Abdel-Khalek, A. A.; Ali, M. M.; Uheida, A.; Muhammed, M.; Zou, X.; Dutta, J. Rare Earth Ions Adsorption onto Graphene Oxide Nanosheets. Solvent Extr. Ion Exch. 2017, 35 (2), 91-103.

https://doi.org/10.1080/07366299.2017.1287509.

(4) Iftekhar, S.; Srivastava, V.; Casas, A.; Sillanpää, M. Synthesis of Novel GA-g-PAM/SiO2 Nanocomposite for the Recovery of Rare Earth Elements (REE) Ions from Aqueous Solution. $J$. Clean. Prod. 2018, 170, 251-259. https://doi.org/10.1016/j.jclepro.2017.09.166.

(5) Iftekhar, S.; Srivastava, V.; Hammouda, S. Ben; Sillanpää, M. Fabrication of Novel Metal Ion Imprinted Xanthan Gum-Layered Double Hydroxide Nanocomposite for Adsorption of Rare Earth Elements. Carbohydr. Polym. 2018, 194, 274-284. https://doi.org/10.1016/j.carbpol.2018.04.054.

(6) Babu, C. M.; Binnemans, K.; Roosen, J. Ethylenediaminetriacetic Acid-Functionalized Activated Carbon for the Adsorption of Rare Earths from Aqueous Solutions. Ind. Eng. Chem. Res. 2018, 57 (5), 1487-1497. https://doi.org/10.1021/acs.iecr.7b04274.

(7) Xiong, C.; He, R.; Pi, L.; Li, J.; Yao, C.; Jiang, J.; Zheng, X. Adsorption of Neodymium(III) on Acrylic Resin (110 Resin) from Aqueous Solutions. Sep. Sci. Technol. 2015, 50 (4), 564-572. https://doi.org/10.1080/01496395.2014.955204. 
